Meta

Journal des traducteurs

Translators' Journal

\title{
La réduction des unités terminologiques complexes de type syntagmatique
}

\section{Tanja Collet}

Volume 42, numéro 1, mars 1997

Lexicologie et terminologie

URI : https://id.erudit.org/iderudit/002348ar

DOI : https://doi.org/10.7202/002348ar

Aller au sommaire du numéro

Éditeur(s)

Les Presses de l'Université de Montréal

ISSN

0026-0452 (imprimé)

1492-1421 (numérique)

Découvrir la revue

Citer cet article

Collet, T. (1997). La réduction des unités terminologiques complexes de type syntagmatique. Meta, 42(1), 193-206. https://doi.org/10.7202/002348ar
Résumé de l'article

Cet article constitue, en quelque sorte, un plaidoyer en faveur d'une étude purement intralinguistique de la réduction des syntagmes terminologiques (ST) au détriment des analyses de type sociohistorique de ce phénomène. La réduction du ST est une manifestation de sa "dynamique discursive". On distingue deux types de réductions : la reprise anaphorique dont les produits ne jouissent d'aucune autonomie contextuelle, et la réduction à caractère lexical qui permet de dériver des ST réduits pouvant transcender le milieu contextuel immédiat. 


\title{
LA RÉDUCTION DES UNITÉS TERMINOLOGIQUES COMPLEXES DE TYPE SYNTAGMATIQUE'
}

\begin{abstract}
Résumé
Cet article constitue, en quelque sorte, un plaidoyer en faveur d'une étude purement intralinguistique de la réduction des syntagmes terminologiques (ST) au détriment des analyses de type sociohistorique de ce phénomène. La réduction du ST est une manifestation de sa «dynamique discursive». On distingue deux types de réductions : la reprise anaphorique dont les produits ne jouissent d' aucune autonomie contextuelle, et la réduction à caractère lexical qui permet de dériver des ST réduits pouvant transcender le milieu contextuel immédiat.
\end{abstract}

\begin{abstract}
In this article, we argue in favor of a purely intralinguistic approach to the study of the reduction of complex terms $(C T)$, and reject any sociohistoric analysis of this phenomenon The reduction of complex terms is one of a number of ways in which a CT interacts with the sentence or sequence of sentences in which it appears, and is an expression of its "discourse dynamics." We've identified two types of reduction: an anaphoric one, the products of which have no contextual autonomy, and a lexical one, the products of which are able to transcend the immediate contextual environment in which they were derived.
\end{abstract}

\section{INTRODUCTION : LA DYNAMIQUE DISCURSIVE DU SYNTAGME TERMINOLOGIQUE}

La phrase, ou mieux encore tout ensemble de phrases, bref le discours, fait et défait le syntagme terminologique (ST). En effet, le ST, qui selon certains trouve sa genèse dans la phrase (thèse transformationnelle de Guilbert (1975)) ${ }^{2}$, interagit, lorsqu'il est actualisé, avec la phrase. Cette dynamique discursive du ST se manifeste sous la forme d'un ensemble de mécanismes intraphrastiques et interphrastiques qui perturbent la linéarité du ST en le rendant discontinu, en le dissolvant ou en effaçant un ou plusieurs de ses constituants. Ces mécanismes discursifs sont au nombre de cinq. Nous distinguons:

(1) la coordination

La coordination met en jeu au moins deux ST et est essentiellement intraphrastique. Les ST joints par coordination n'ont pas à appartenir à la même matrice. Cependant, une identité soit au niveau du déterminé (dé) soit au niveau du déterminant (dã) est nécessaire. La coordination se caractérise par l'effacement du dé ou du dã commun aux ST qu'elle joint. Plus précisément, selon qu'il y a identité du dé ou du dã, la coordination de deux ST se réalise par l'effacement du dé commun du deuxième ST ou, le cas échéant, par l'effacement du dã commun du premier ST et par l'insertion d'un joncteur, généralement ET, OU ou une virgule.

Exemple: bande de réception et bande d'émission $\rightarrow$ bandes de réception et d'émission ( $«$ La fréquence de l'oscillateur local est fixée par la différence des fréquences des bandes de réception et d'émission.» (Maral 1982: 104)) 
(2) la prédication ${ }^{3}$

La prédication met en jeu un seul ST et est essentiellement intraphrastique. Elle dissout le ST en un sujet et un prédicat, tout en respectant la binarité ${ }^{4}$ du ST. En effet, le dé est incorporé au sujet et le dã, précédé du verbe, le plus souvent la copule être, au prédicat de la phrase.

Excmple : (répćtcur ${ }_{d e ́}$ à double changement de fréquence $\left.{ }_{\mathrm{dã}}\right)_{\mathrm{ST}} \rightarrow$ (répéteur $_{\text {sujet }}$ être à double changement de fréquence $\left.{ }_{\text {prédicat }}\right)_{\mathrm{Ph}}$, ou sous forme d'une arborescence :

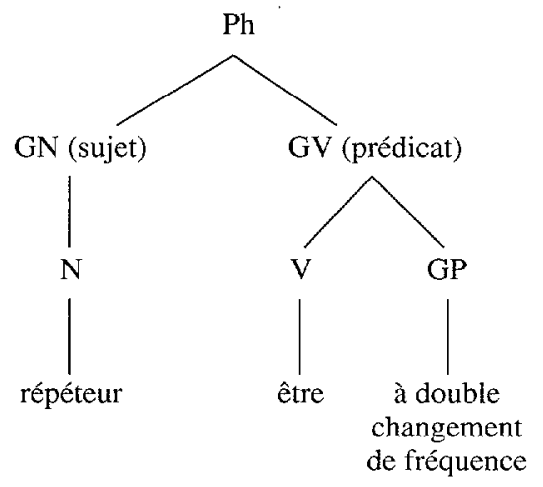

(«Pour les deux modules $\mathrm{A}$ et $\mathrm{B}$, les répéteurs sont à double changement de fréquence.» (CNES/CNET 1983, t. 3:288))

(3) l'insertion

L'insertion met en jeu un seul ST et est essentiellement intraphrastique. Elle respecte la binarité du ST, l'élément inséré ( $\mathrm{Adj}$, Adv, etc.), venant se placer le plus souvent entre le dé et le dã. L'élément inséré ne correspond pas à un trait pertinent distinctif de la notion dénommée par le ST.

Exemple: antenne du type Cassegrain $\longleftarrow$ antenne Cassegrain («Pour des antennes du type Cassegrain la figure 8.12 donne le diamètre de l'antenne en fonction de l'ouverture à $3 \mathrm{~dB}$ pour les fréquences $4 \mathrm{GHz}$ et $12 \mathrm{GHz}$ » (Maral 1982: 239))

(4) la dénomination

La dénomination met en jeu un seul ST et est essentiellement intraphrastique. Elle respecte la structure binaire du ST, l'élément inséré (dit, appelé, etc.), venant se placer généralement entre le dé et le dã.

Exemple : amplificateur dit chaud $\leftarrow$ amplificateur chaud $(\ll[\ldots]$ mais la cryogénie était liée à un matériel complexe dont on s'est libéré dès que les températures de bruit des amplificateurs dits "chauds» ont été suffisamment abaissées pour n'entraîner qu'un léger agrandissement du diamètre de l'antenne.» (CNES / CNET 1983, t. 3: 38))

Nous distinguons la dénomination de l'insertion. La dénomination se caractérise par deux contraintes: une au niveau de la catégorie grammaticale de l'élément inséré, laquelle est 
le plus souvent un $A d j_{\text {participe passé, et une au niveau du champ sémantique de l'élément }}$ inséré, lequel correspond nécessairement au champ de DÉNOMMER. Par contre, l'insertion se caractérise par l'absence de contrainte au niveau du champ sémantique de l'élément inséré - il y a cependant, comme il a été mentionné ci-dessus, une contrainte au niveau notionnel - et par la diversité des catégories grammaticales admises.

\section{(5) la réduction}

La réduction met en jeu un seul ST, et une phrase ou plusieurs phrases plus ou moins espacées dans le texte. La réduction procède à l'effacement en entier d'un ou de plusieurs constituants du ST. Des mécanismes discursifs présentés ci-dessus, le mécanisme réducteur est le seul à pouvoir affecter de façon permanente la terminologie d'un domaine de spécialité et, de ce fait, il a particulièrement retenu notre attention.

Exemple: service mobile aéronautique par satellite $\Longleftrightarrow$ service aéronautique par satellite $\Longleftrightarrow$ service aéronautique («3. LE SERVICE MOBILE AÉRONAUTIQUE PAR SATELLITE [...] Pour la surveillance des zones dépourvues d'infrastructure, le service aéronautique par satellite permet d'assurer une surveillance de la zone survolée de façon permanente. [...] Au-delà du service aéronautique propre il peut être envisagé une extension des services de transmission de données et de téléphonie à l'usage des passagers.» (CNES / CNET 1983, t. 3: 215-218))

Dans cet article, il sera donc plus particulièrement question de la réduction. Mais avant d'entrer réellement en matière, il convient de bien définir le ST, c'est-à-dire l'unité terminologique complexe sujette à la réduction ainsi qu'aux autres mécanismes discursifs que nous venons de décrire très brièvement. Nous proposons la définition ${ }^{5}$ suivante: Le syntagme terminologique est une unité complexe qui :

(i) est formée selon les règles de la syntaxe;

(ii) est composée d'au moins deux unités graphiquement simples;

(iii) a une structure interne binaire, constituée d'un déterminé et d' un déterminant;

(iv) se caractérise par un degré de figement relatif;

(v) a le statut de terme, c'est-à-dire de dénomination d'une notion comprise dans le système notionnel d'un domaine.

Cette définition privilégie les ST subordonnés ou endocentriques (dé exprimé) aux dépens des ST coordonnés ou exocentriques (dé non exprimé). Les ST subordonnés se caractérisent par une binarité structurelle (dé dã) qui traduit une binarité notionnelle sousjacente (hyperonymie, hyponymie), en ce sens que le dé identifie l'hyperonyme, tandis que le dã range le ST dans l'ensemble des hyponymes de cet hyperonyme, exemple :

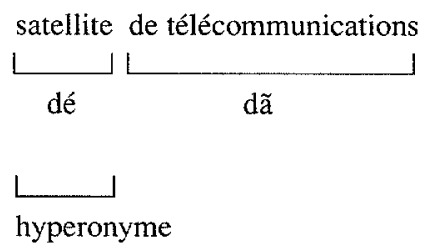

hyponyme 
Les ST coordonnés ou exocentriques, par contre, n'ont pas une structure binaire pouvant être analysée comme la suite dé dã. Leurs constituants, cependant, peuvent être considérés comme représentant le dã d'un dé non exprimé, exemple:

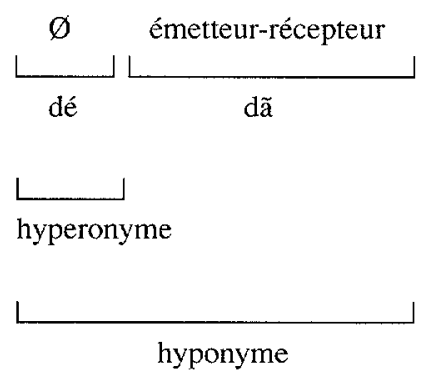

Les ST coordonnés sont exclus de la définition car ils semblent résister à la mise en œuvre des mécanismes de la dynamique discursive. C'est, à tout le moins, ce qui semble ressortir des textes technoscientifiques ${ }^{6}$ que nous avons analysés. En nous basant donc sur ces données ainsi que sur les exemples fournis par Guilbert (1975), Duquet-Picard (1986), Portelance (1989), et Kocourek (1991), nous nous permettons de conclure, jusqu'à preuve du contraire, que seuls les ST subordonnés sont sujets aux mécanismes de la dynamique discursive. En effet, seuls les ST subordonnés semblent tolérer la réduction (ex. antenne à réflecteur parabolique $\Longleftrightarrow$ antenne parabolique). Feyry-Miannay (1984:41) soupçonne, cependant, que bon nombre des ST coordonnés sont issus de la réduction d'un ST subordonné par effacement du terme de base, c'est-à-dire du dé (ex. circuit échantillonneurbloqueur $\Longleftrightarrow$ échantillonneur-bloqueur). À la base d'un tel ST coordonné se trouve donc un ST dont les constituants se trouvent dans un rapport de co-subordination? ${ }^{7}$. En effet, dans l'exemple ci-dessus, échantillonneur et bloqueur sont subordonnés en tant qu'unité (dã) au terme de base (dé) circuit qu'ils qualifient et qui sera élidé.

\section{LA RÉDUCTION}

Nous soutenons que la réduction du ST, c'est-à-dire la modification de sa suite linéaire par l'effacement en entier d'un ou de plusieurs de ses constituants, est une manifestation observable de la dialectique qui s'installe d'emblée, lors de l'actualisation du ST, entre son caractère analytique d'une part, et les exigences de l'économie verbale (cohésion et cohérence textuelles) d'autre part. Un petit nombre d'auteurs (tels Guilbert (1970) et (1975), Lotte (1981), Alber-Dewolf (1984), Duquet-Picard (1986), Portelance (1989), et Kocourek (1991)) font allusion au phénomène de la réduction. Toutefois, les analyses sommaires de ces auteurs n'associent pas la réduction ${ }^{8}$ des ST au phénomène de la variation contextuelle et, par conséquent, ne démontrent pas la dynamique discursive de ce mécanisme.

Ainsi, certains auteurs se servent tantôt de critères sémantiques, tantôt de critères sociohistoriques, donc extralinguistiques, dans l'explication de la réduction des ST. Voici un exemple d'une telle analyse, tiré de Guilbert (1975:273-274):

Le type d'abréviation de composé le plus répandu ${ }^{9}$ consiste à réduire le composé formé du syntagme $\mathrm{N}+$ adj. au second terme adjectif : [...], une voiture automobile $>$ une automobile [...] On remarque que le terme maintenu est le déterminant [...] Le pouvoir de désignation se situe dans le déterminant et non dans la base à valeur générale. Cette vocation du déterminant à représenter le syntagme découle du fait de sa fonction et non de sa place. [...] Il 
peut arriver que l'unité syntagmatique de forme nom + adj. se réduise à la base : voiture autornobile $>$ voiture. L'abréviation n'est plus, alors, d'essence seulement syntagmatique; son apparition implique que l'évolution intervenue sur le plan du référent interdise toute ambiguitté, le mode de locomotion le plus répandu étant celui de l'automobile.

Portelance (1989: 146) rejoint la pensée de Guilbert dans son analyse du rapport reliant cigarette et cigarette filtre.

La cigarette filtre s'oppose à la cigarette à bout uni (ou sans filtre). Lors de l'apparition de ce référent sur le marché de consommation, il n'y a que la cigarette sans filtre que l'on nomme tout simplement cigarette. On introduit ensuite une deuxième cigarette avec comme particularité l'ajout d'un bout filtre: cigarette devient alors une appellation générique qui regroupe deux référents : cigarette à bout uni et cigarette à bout filtre. Les habitudes de consommation évoluant, le deuxième type de cigarette devient le produit le plus répandu, si bien que c'est souvent le générique qui le désigne alors qu'il faut apporter des précisions lorsque l'on désire une cigarette sans filtre, expression qui remplace maintenant dans l'usage cigarette à bout uni. On dit même des sans filtre.

Portelance (1989: 195) définit la réduction comme la suppression d'une information devenue non différentielle.

Chaque détermination ${ }^{10}$ [d'un syntagme terminologique] constitue une caractéristique différentielle, une information, qui, ajoutée à un noyau, identifie une notion parmi un ensemble de notions. Lorsque la caractéristique n'est plus différentielle, la détermination devenant inutile tombe. Le mécanisme en jeu dans la réduction des syntagmes s'articule à partir d'un système vectoriel implicite/explicite ${ }^{11}$.

Bien que ces analyses de type sociohistorique, qui supposent que la réduction des ST est conditionnée par les évolutions sur le plan du référent, soient valides dans certains cas, elles ne permettent de rendre compte que d'une infime partie des ST réduits et s'avèrent insuffisantes voire inadéquates pour rendre compte de la variation contextuelle, phénomène synchronique par excellence, le mécanisme réducteur que nous étudions se déroulant à l'intérieur du texte. Considérons les paragraphes suivants :

Le cas considéré est celui du service mobile maritime par satellite qui a pour objet d'assurer des télécommunications entre les navires et la terre par l'intermédiaire de satellites; à terre, les liaisons par satellite aboutissent ou partent de «stations côtières» qui assurent la connexion avec le réseau général de télécommunications. Le premier système de ce service à être mis en place utilise les satellites MARISAT des États-Unis. (CNES / CNET 1982, t. 1:250)

Dans les deux cas, le problème de l'accès au satellite se présente très différemment selon qu'on le considère vis-à-vis des stations terriennes de navire ou des stations terriennes côtières. On peut prévoir que le nombre de stations de navire sera très élevé (plusieurs milliers), alors que celui des stations côtières sera faible (au maximum quelques dizaines par océan). (CNES/CNET 1982 t. $1: 251$ )

Le ST service mobile maritime par satellite, qui apparaît sous sa forme pleine dans la première ligne du premier paragraphe, réapparaît sous sa forme minimale, service, précédée du déictique $c e$, dans la dernière ligne de ce même paragraphe. Le ST station côtière du premier paragraphe réapparaît sous sa forme pleine station terrienne côtière dans le deuxième paragraphe. Ce même paragraphe contient aussi la forme réduite station côtière. Le ST station terrienne de navire se réduit dans le deuxième paragraphe à station de navire. Dans les deux paragraphes, formes pleines et réduites de trois ST sont employés côte à côte, phénomène dont l'analyse sociohistorique ne peut rendre compte.

Nous préconisons une explication purement intralinguistique de la réduction, et jugeons qu'une telle approche cerne mieux la réalité textuelle où formes pleines et réduites 
d'un même ST se côtoient. Nous établissons une distinction entre la réduction à caractère lexical (RL) pouvant transcender le milieu contextuel immédiat (ex. station terrienne de navire $\Longleftrightarrow 12$ station de navire, station terrienne côtière $\Longleftrightarrow 13$ station côtière), et la reprise anaphorique (RA), qui est purement contextuelle (ex. service mobile maritime par satellite $\Longleftrightarrow$ service). Cette distinction entre RA et RL est largement acceptée parmi les auteurs, mais rarement motivée. Nous la retrouvons chez Alber-Dewolf (1984) (ellipse contextuelle vs ellipse néonymique), Duquet-Picard (1986) (ellipse contextuelle vs ellipse non contextuelle) et chez Kocourek (1991) (ellipse contextuelle vs ellipse lexicale). Dans La créativité lexicale (1975), Guilbert s'est surtout consacré à l'analyse de la RL; auparavant, cependant, dans La dérivation syntagmatique dans les vocabulaires scientifiques et techniques (1970: 119), il avait fait brièvement allusion à la RA.

L'opposition entre la dérivation (ou composition) lexématique et la dérivation syntagmatique réside dans le fait que le noyau de l'unité synaptique ${ }^{14}$ garde son autonomie en tant qu'élément disjoint. Cette liberté se traduit par le fait qu'il sert d'élément anaphorique, de pronom pour l'ensemble de l'unité synaptique complexe (cette charrue) reprend (la charrue pour labour à plat à traction animale sans avant-train) alors que le lexème construit formalisation ne peut être repris que par la même unité répétée (cette formalisation) ou par le pronom elle (la-lui).

Portelance (1989), pour sa part, semble ne définir que la RL. Enfin, Lotte (1981), en dressant les principes d'établissement d'une terminologie scientifique et technique, juge primordiale l'indépendance contextuelle ${ }^{15}$ du terme. Celui-ci ne peut être influencé par la phrase dans laquelle il est employé, ni sur le plan notionnel ni sur le plan dénominationnel16. Lotte (1981: 7) prévoit cependant deux exceptions qui concernent la reprise anaphorique.

Un écart par rapport au principe de l'indépendance du terme à l'égard du contexte n'est permis que dans deux cas.

1. Lorsqu'on sait de quelle notion il s'agit, on peut omettre les éléments déterminants du terme. Si un livre est consacré en entier aux moteurs à carburateur, il est inutile de répéter ce terme à chaque fois; on peut se contenter du terme générique «moteur».

2. L'utilisation répétée mais nécessaire du terme générique peut entraîner des lourdeurs stylistiques; il convient alors de rejeter les éléments déterminants du terme et de les remplacer normalement par les démonstratifs correspondants (ce, cette; le, la... en question, etc.).

Dans ces cas, il n'y a pas de changement de la signification ou de la forme du terme, mais un simple remplacement de la notion spécifique par la notion générique. ${ }^{17}$

Il semble donc que la plupart des auteurs reconnaissent explicitement ou implicitement l'existence de deux types de réductions, notamment la RA et la RL, à l'exception de Lotte et de Portelance qui ne semblent reconnaître que la RA ou la RL respectivement. Cette distinction, reconnue mais rarement motivée, repose, selon nous, sur un ensemble de critères fonctionnels, onomasiologiques, formels, sémantiques et discursifs, ainsi que sur les différences au niveau de la régularité des mécanismes de la RA et de la RL.

Mentionnons d'abord que la fonction principale de la RA est d'établir des liens entre les phrases d'un texte, donc de contribuer à la cohésion et à la cohérence textuelles. La RL, assurant dans une certaine mesure cette même fonction, se distingue de la RA par sa capacité de créer des variantes terminologiques, susceptibles de devenir des membres permanents de la terminologie du domaine du ST plein. Nous jugeons que les produits de la RL sont des variantes et non des synonymes et préférons donc distinguer la variation contextuelle de la synonymie. La variation contextuelle est l'emploi simultané dans un texte de plusieurs variantes d'un même terme, en l'occurrence d'un ST. Les variantes résultent de la réduction du ST. Elles ont donc toutes un même signifié mais des signifiants différents, en ce sens qu'il y a variation du nombre de constituants, lesquels doivent nécessairement faire partie de la forme pleine du ST. Considérons les variantes 'service 
mobile aéronautique par satellite' $\Longleftrightarrow$ 'service aéronautique par satellite' $\Longleftrightarrow$ 'service aéronautique' qui se trouvent liées par un rapport réductionnel. Par contre, la synonymie suppose en règle générale des étymons différents. En effet, les ST synonymes se caractérisent par la présence de constituants non identiques au niveau du dé, au niveau du dã, ou au niveau à la fois du dé et du dã. Considérons les exemples suivants tirés de Guilbert (1967: 266): «vol cosmique/vol spatial [non-identité du dã], base de lancement/aire de lancement [non-identité du dé], voyage interplanétaire/vol spatial [non-identité du dé et du dã]».

Au niveau onomasiologique, la RA et la RL se distinguent par leur «contextualité» (autonomie/dépendance contextuelle), qui semble être fonction d'une éventuelle transgression, au niveau dénominationnel, de la hiérarchie onomasiologique ${ }^{18}$. En effet, les ST réduits, qui ne cö̈ncident pas formellement avec un hyperonyme et qui conservent ainsi le niveau onomasiologique du ST plein même hors contexte, sont issus du mécanisme réducteur à caractère lexical. Par contre, les ST réduits, qui coïncident formellement avec un hyperonyme et qui, par conséquent, ne conservent le niveau onomasiologique du ST plein qu'en contexte, sont des RA ${ }^{19}$. Nous proposons de considérer le ST plein et ses formes réduites comme les membres d'un paradigme réductionnel, que l'on pourrait représenter comme suit :

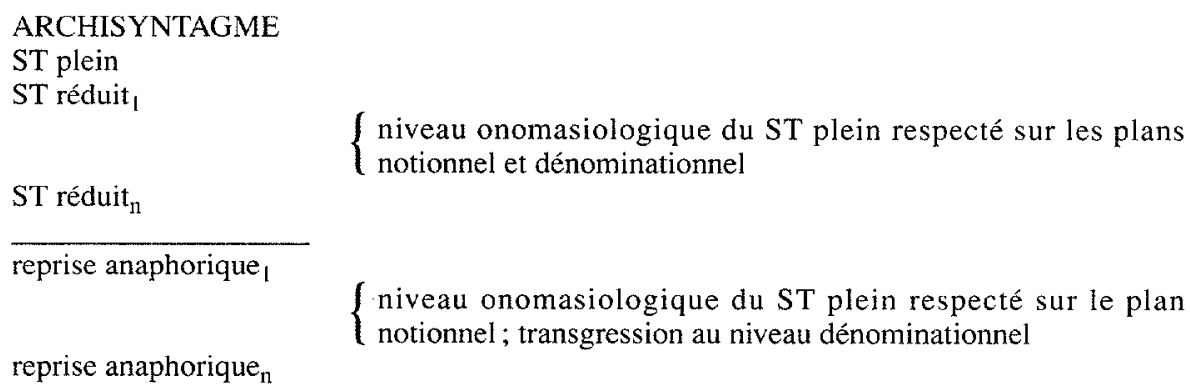

$\{$ niveau onomasiologique du ST plein respecté sur les plans \{ notionnel et dénominationnel

$\{$ niveau onomasiologique du ST plein respecté sur le plan notionnel; transgression au niveau dénominationnel

Les membres d'un paradigme réductionnel doivent se situer tous au même niveau onomasiologique, c'est-à-dire qu'ils doivent tous respecter le niveau onomasiologique du ST plein. Les produits de la RL respectent le niveau onomasiologique du ST plein sur les plans notionnel et dénominationnel. Nous postulons que leur caractère lexical découle, justement, de ce respect des deux plans notionnel et dénominationnel. Considérons la série hiérarchique suivante:

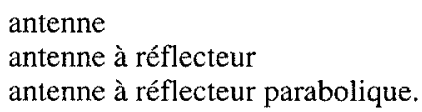

Dans cette série, antenne est l'hyperonyme de antenne à réflecteur qui, à son tour, est l'hyperonyme de antenne à réflecteur parabolique. Antenne à réflecteur parabolique est réductible en contexte, et se réduit à antenne parabolique et non à *antenne à réflecteur. La forme réduite *antenne à réflecteur semble inadmissible car elle entre en conflit avec le syntagme plein antenne à réflecteur, qui est l'hyperonyme du syntagme plein antenne à réflecteur parabolique dont *antenne à réflecteur constitue la variante réduite. Nous obtenons, donc, la série hiérarchique suivante:

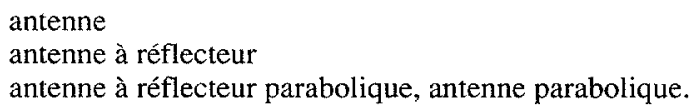


Les produits de la RA, par contre, se caractérisent par une transgression du niveau onomasiologique sur le plan dénominationnel, et sont, par ce fait même, liés au contexte dans lequel ils apparaissent. Du point de vue notionnel, donc, ils ne peuvent conserver le niveau onomasiologique du ST plein que dans le contexte dans lequel la RA s'est produite. Ainsi, antenne à réflecteur parabolique se réduit dans le cas d'une RA à son déterminé antenne qui coïncide du point de vue dénominationnel avec l'hyperonyme antenne.

Sur le plan formel, la RA et la RL se distinguent par les types d'effacements admis. La $\boldsymbol{R A}$ n'admet que l'effacement en entier du dã, simple ou complexe, c'est-à-dire syntagmatique, du ST, et conserve son dé partiellement ou en entier. La préservation partielle du dé n'est permise que lorsque ce dernier constitue lui-même un ST subordonné. Il s'ensuit qu'un ST à dé syntagmatique peut avoir au moins deux RA, notamment le dé syntagmatique et le dé de celui-ci obtenu par effacement de son dã. Ce qui précède peut être représenté sous forme de schéma comme suit:

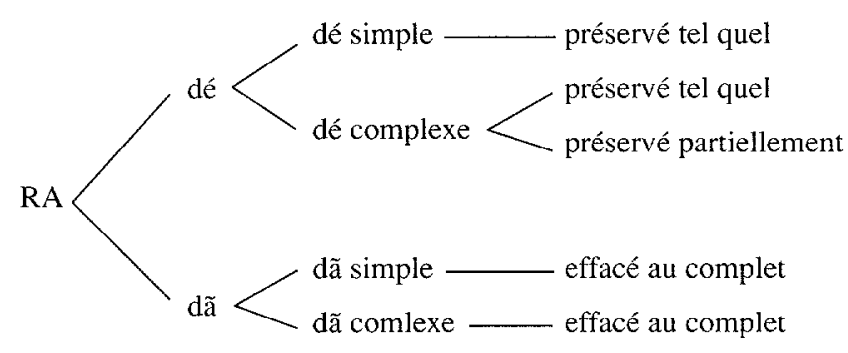

Exemples:

- détecteur dé solaire $_{\mathrm{dã}} \longleftrightarrow$ détecteur (dé simple préservé tel quel);

- station terrienne $\mathrm{dé}_{\text {fixe }} \mathrm{dãa}_{\mathrm{a}} \Longleftrightarrow$ station terrienne (dé complexe préservé tel quel);

- panneau solaire dé orientable $e_{\mathrm{d} a ̃} \Longleftrightarrow$ panneau (dé complexe préservé partiellement);

- satellite $_{\text {dé géostationnaire }}$ dã $\Longleftrightarrow$ satellite (dã simple effacé au complet);

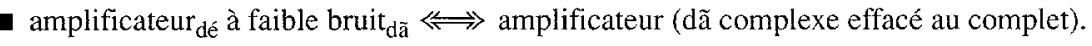

La $R \boldsymbol{L}$, par contre, permet l'effacement tantôt des éléments de servitude syntaxique (prépositions, articles) tantôt des éléments forts. L'effacement des éléments de servitude syntaxique concerne les prépositions et/ou articles introduisant le dã ou faisant partie intégrante de celui-ci. Dans le cas des éléments forts, l'effacement porte tantôt sur le dé, tantôt sur le dã. L'effacement du dé, simple ou complexe, ou du dã, simple ou complexe, peut être partiel ou total. L'effacement d'éléments forts peut, dans certains cas, entraîner la chute des éléments de servitude syntaxique, voire entraîner un changement de catégorie grammaticale. En effet; pour être grammaticaux, les produits de la RA et de la RL doivent appartenir à la même catégorie grammaticale que le ST ${ }^{20}$ plein à partir duquel ils ont été obtenus. Alors que cette contrainte est rarement problématique pour une RA, celle-ci conservant toujours le dé, elle peut l'être pour une RL. La contrainte de la préservation de la catégorie grammaticale peut, dans ce cas, entraîner un changement de la catégorie grammaticale du constituant non effacé :

ex. N Adj $\Longleftrightarrow A$ d $_{\text {nominalisé }}$ onde porteuse $\Longleftrightarrow$ porteuse, 
ou, par exemple, entraîner la chute de la préposition introduisant le dã après effacement du dé:

ex. $\mathrm{N}_{1}$ Prép $\mathrm{N}_{2} \Longleftrightarrow \mathrm{N}_{2}$ : système d'entraînement $\Longleftrightarrow$ entraînement.

L'effacement d'éléments forts entraîne également la chute des éléments de servitude syntaxique et/ou un changement de catégorie grammaticale lorsque le résultat risque de coïncider sinon avec une suite agrammaticale :

ex. $N_{1}$ Prép $N_{2}$ Adj $\Longleftrightarrow N_{1}$ Adj et $* \Longleftrightarrow N_{1}$ Prép Adj: détecteur d'horizon terrestre $\Longleftrightarrow$ détecteur terrestre et $* \Longleftrightarrow$ détecteur de terrestre

ex. $N_{1}$ Prép ArtDéf $N_{2}$ Adj $\Longleftrightarrow N_{1}$ Prép ArtDéf Adjnominalisé et $* \Longleftrightarrow N_{1}$ Prép ArtDéf

Adj : orbite des satellites géostationnaires $\Longleftrightarrow$ orbite des géostationnaires.

Ce qui précède peut être représenté sous forme de schémas comme suit:

Effacements d'éléments de servitude syntaxique

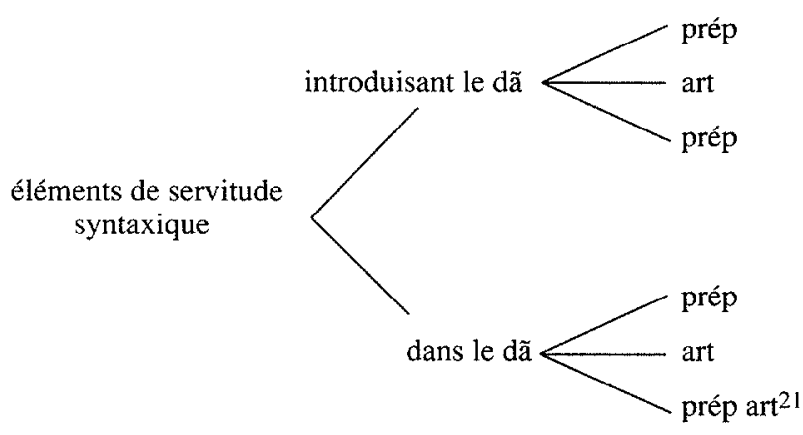

${ }^{2 !}$ Exemples :

- axe $\mathrm{dé}_{\mathrm{de}}$ de lacet $\mathrm{d}_{\mathrm{d} a} \Longleftrightarrow \mathrm{axe}_{\mathrm{dé}}$ lacet $_{\mathrm{d} \tilde{a}}$ (effacement prép introduisant dã);

- stabilisation $\mathrm{dé}$ sur les trois axes $\mathrm{dã} \Longleftrightarrow$ stabilisation $_{\mathrm{dé}}$ sur trois axes $\mathrm{dã}_{\text {(effacement art }}$ introduisant dã);

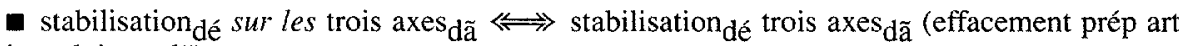
introduisant dã);

- rapport $\mathrm{dé}_{\mathrm{d}}$ de la puissance $d e$ porteuse à la puissance de bruit $\mathrm{d}_{\tilde{d} a} \Longleftrightarrow$ rapport $_{\mathrm{dé}}$ de la puis-

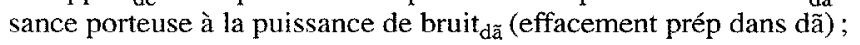

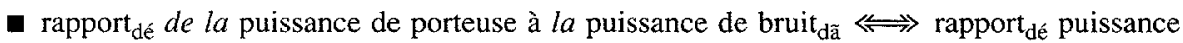
de porteuse à puissance de bruit ${ }_{\text {dã }}$ (effacement art dans dã et prép art introduisant dã);

- rapport $\mathrm{dé}_{\text {de }}$ de la puissance de la porteuse à la densité spectrale de bruit $\mathrm{d}_{\mathrm{daz}} \Longleftrightarrow$ rapport $_{\mathrm{dé}}$ de

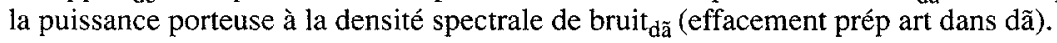




\section{Effacements d'éléments forts au niveau du dé}

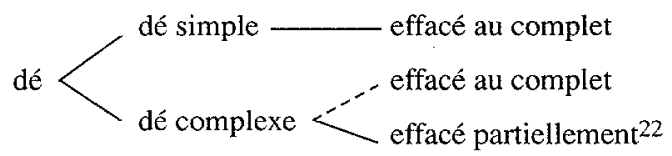

${ }^{22}$ Exemples:

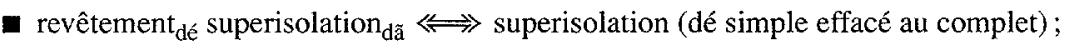

- matériau dé diélectrique $_{\mathrm{dã}} \Longleftrightarrow$ diélectrique (effacement complet du dé simple avec nominalisation subséquente du dã adjectival préservé);

- phase $_{\mathrm{dé}}$ de transfert $\mathrm{dã} \Longleftrightarrow$ transfert (effacement complet du dé simple avec chute subséquente de la prép introduisant le dã);

- panneau solaire dé déployable $_{\mathrm{da}} \Longleftrightarrow$ panneau déployable (effacement partiel du dé complexe);

- station terrienne $\mathrm{dé}_{\mathrm{d}}$ de navire $\mathrm{dã}_{\mathrm{da}} \longleftrightarrow$ station de navire (effacement partiel du dé complexe).

Effacements d'éléments forts au niveau du dã

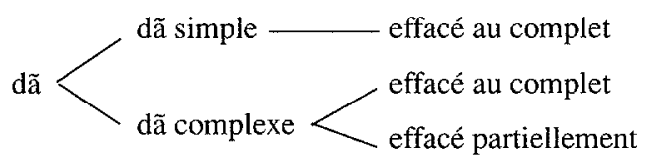

Exemples:

- navette $_{\text {dé }}$ spatiale $_{\mathrm{dã}} \longleftrightarrow$ navette (dã simple effacé au complet);

- bande $_{\text {dé de fréquences }}$ dã $\Longleftrightarrow$ bande (effacement complet du dã simple avec chute subséquente de la prép introduisant le dã);

- batterie dé d'accumulateurs électrochimiques $_{\text {dã }} \Longleftrightarrow$ batterie (effacement complet du dã complexe avec chute subséquente de la prép introduisant le dã);

- détecteur ${ }_{\text {dé }}$ d'horizon terrestre dã $\Longleftrightarrow$ détecteur terrestre (effacement partiel du dã complexe avec chute subséquente de la prép introduisant le dã);

- orbite $_{\mathrm{dé}}$ des satellites géostationnaires $\mathrm{dã} \Longleftrightarrow$ orbite géostationnaire (effacement partiel du dã complexe avec chute subséquente des éléments de servitude syntaxique (prép art));

m orbite $_{\text {dé des satellites géostationnaires }}$ dã $\Longleftrightarrow$ orbite des géostationnaires (effacement partiel du dã complexe avec nominalisation subséquente de l'adj (dã du dé du dã syntagmatique) préservé);

- phase $_{\text {dé d'orbite de transfert }}$ dã $\Longleftrightarrow$ phase de transfert (effacement partiel du dã complexe avec chute subséquente de la prép introduisant le dã).

Sur le plan sémantique, la RL semble produire un double mouvement. En effet, au niveau du terme, le syntagme plein et ses variantes réduites ont le même signifié mais des 
signifiants différents, tandis qu'au niveau des constituants des variantes réduites, il y a redistribution de la charge sémantique des constituants du syntagme plein et préservation des signifiants non supprimés. Il semble donc que la RL a pour effet de modifier la nature compositionnelle du signifié du syntagme plein. Considérons la paire antenne à réflecteur parabolique $\Longleftrightarrow$ antenne parabolique, qui au niveau du terme ont un même signifié ils identifient une même notion -, mais des signifiants différents. Au niveau des constituants, cependant, nous constatons que le signifié d'antenne à réflecteur parabolique est plutôt compositionnel, en ce sens qu'il se laisse aisément déduire des signifiés des constituants et des relations syntaxiques qui les régissent. Le signifié du syntagme réduit antenne parabolique, par contre, nous semble moins compositionnel, l'adjectif parabolique ne qualifiant pas tout à fait antenne. Une antenne parabolique n'est pas une antenne qui est parabolique, mais une antenne dotée d'un réflecteur parabolique. Cette modification de la nature compositionnelle du signifié du ST a pour conséquence d'augmenter sa cohésion interne. Les variantes réduites se caractérisent, donc, par une plus grande cohésion par rapport au syntagme plein. La RA, cependant, a pour effet d'augmenter la cohésion et la cohérence textuelles au détriment de la cohésion interne du syntagme plein qu'elle semble annuler.

Sur le plan discursif, l'écart phrastique maximal entre le syntagme plein et ses variantes anaphoriques semble moins important que l'écart phrastique maximal susceptible de séparer un syntagme plein et sa variante obtenue par RL. Nous ne disposons cependant pas encore de statistiques à ce propos.

La RA et la RL se distinguent aussi par la régularité de leurs procédés. En effet, la RA semble pouvoir frapper tout ST subordonné actualisé. Cette régularité de la RA est encore renforcée par la régularité de ses règles réductionnelles que nous présumons, pour le moment, être seulement au nombre de deux (notamment une règle simple et une à deux volets) et qui procèdent toutes les deux à un effacement complet du dã du ST plein quelle que soit sa matrice. Il s'agit des règles suivantes:

1) [Dé Dã $]_{\mathrm{ST}} \Longleftrightarrow[\text { Dé } \varnothing]_{\mathrm{RA}}$

2a) [[dé dã $\left.]_{\mathrm{Dé}} \mathrm{Da}\right]_{\mathrm{ST}} \Longleftrightarrow\left[[\text { dé dã }]_{\text {Dé }} \emptyset\right]_{\mathrm{RA}}$

2b) [[dé dã $]_{\text {Dé }}$ Dã $\left.]_{S T} \Longleftrightarrow[\text { [dé } \emptyset]_{\text {Dé }} \varnothing\right]_{\mathrm{RA}}$

La RL, par contre, paraît plus sélective et semble affecter tel ST plutôt que tel autre; un lien déclencheur pouvant exister entre la $\mathrm{RL}$, les caractéristiques sémantiques et /ou syntaxiques des constituants du ST et sa matrice. La RL se caractérise également par une grande variété au niveau de ses règles réductionnelles. Nous ne disposons malheureusement pas encore de la liste des règles réductionnelles de la RL. Il semble nécessaire, dans un premier temps, de lister les règles réductionnelles par la matrice du syntagme plein avant de procéder à toute unification et simplification des règles. Voici, en guise d'exemple, les règles réductionnelles des matrices $\mathrm{N}$ Adj et $\mathrm{N}_{1}$ Prép $\mathrm{N}_{2}$ :

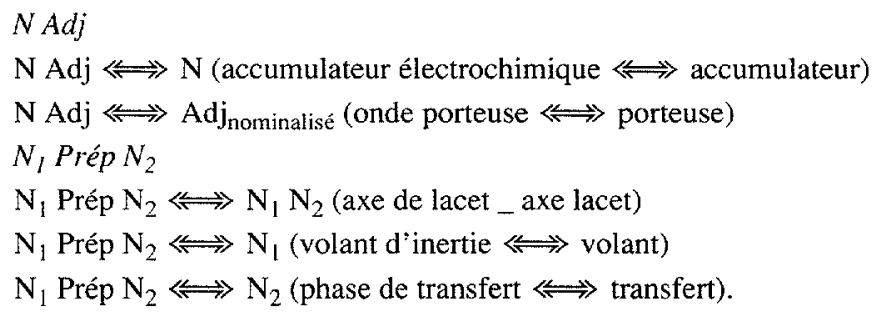




\section{CONCLUSION}

La réduction, mécanisme discursif ne s'exerçant que sur les ST actualisés, paraît aller à l'encontre du principe wüstérien de la bi-univocité, principe terminologique formulé dans un cadre théorique visant la normalisation. En effet, la RA semble produire un effet homonymique, en ce sens qu'en contexte la forme du générique ou de l'hyperonyme (ex. antenne) peut s'approprier le signifié de ses spécifiques ou hyponymes (cx. antenne à réflecteur, antenne à réflecteur parabolique, antenne cornet, antenne à couverture globale, etc.). Cela peut être représenté comme suit:

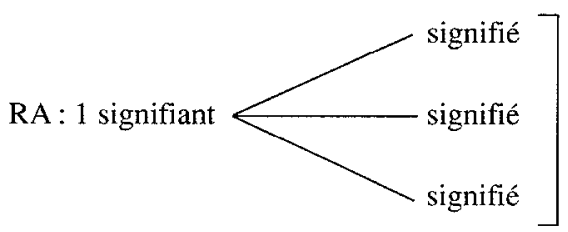

en contexte

La RL, par contre, semble produire un effet synonymique en ce sens que plusieurs signifiants, obtenus par réduction du signifiant du syntagme plein, ont un même signifié. Cela peut être représenté comme suit:

RL: 1 signifté

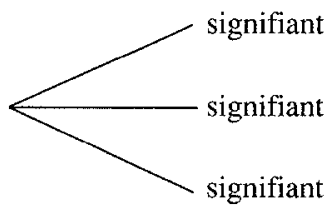

Mentionnons, pour terminer, qu'à l'opposé de Guilbert (1965:275), nous soutenons que le ST, même celui qui a atteint un haut degré de lexicalisation, se caractérise par une instabilité fondamentale du rapport syntagmatique au plan du discours.

Le passage du statut de groupement syntagmatique du discours au statut d'unité lexicale suppose la réalisation d'un certain nombre de conditions: la stabilité du rapport syntagmatique au plan du discours, la stabilité du rapport de signification entre l'unité syntagmatique et un signifié unique, la fréquence d'emploi qui stabilise à la fois le lien syntagmatique et le rapport de signification. 
Cette instabilité syntagmatique du ST subordonné actualisé est fonction de sa fréquence d'emploi. En effet, c'est justement la répétition du ST qui met en branle l'économie verbale et, de ce fait, déclenche les mécanismes de la réduction tant du type anaphorique que du type lexical.

\section{Notes}

1. Cette étude est subventionnée par le Conseil de recherches en sciences humaines du Canada (CRSH) par le biais de la bourse 752-93-1132.

2. «Le principe de formation de l'unité lexicale synaptique réside, selon l'optique générativiste, dans une relation de détermination issue d'une phrase prédicative dont les éléments sont un syntagme nominal fournissant la base de la formation et un syntagme verbal générant le déterminant :

$\begin{array}{ccc}\text { Avion à réaction } & \mathrm{SN} & \mathrm{SV} \\ \text { [L'avion] } & \text { [est à réaction] }\end{array}$

La transformation s'opère par la relativisation du syntagme verbal : l'avion [qui est] à réaction, et par l'effacement du relatif et de la copule.» (Guilbert $1975: 253$ )

3. La prédication constitue, en quelque sorte, le mouvement inverse du phénomène créateur de ST décrit par Guilbert (1975: 253). Elle est ultérieure à ce moment de création, et ne concerne que le ST lexicalisé ou en voie de lexicalisation.

4. Par binarité, nous entendons le fait que la structure interne du ST se laisse ramener à la suite dé dã, et plus rarement à la suite dã dé.

5. Il s'agit d'une définition conçue spécifiquement pour le ST français.

6. Les textes analysés appartiennent tous au domaine des télécommunications spatiales.

7. Alber-DeWolf $(1984: 214)$ définit ainsi le rapport de co-subordination : «Il y a rapport de co-subordination quand deux ou plusieurs constituants d'une UTC [unité terminologique complexe] sont coordonnés pour former une unité de même niveau notionnel, unité qui peut être subordonnée à une autre ou à laquelle une autre unité peut être subordonnée.»

8. Nous distinguons deux types de réduction : la reprise anaphorique et la réduction à caractère lexical. Cette remarque concerne surtout la réduction à caractère lexical.

9. Nous ne disposons malheureusement pas encore de statistiques, mais à première vue nos données empiriçues semblent aller à l'encontre de cette affirmation.

10. Détermination $=$ dã et noyau $=$ dé dans notre terminologie.

11. La définition, telle que formulée par Portelance, est trop restrictive et ne permet pas de rendre compte du mécanisme réducteur qui ne conserve que le dã et supprime le dé (ex. voiture automobile $\Longleftrightarrow$ automobile, matériau diélectrique $\Longleftrightarrow$ diélectrique).

12. La flèche à double tête est à la fois le signe d'une mise en relation et le reflet de la réalité textuelle où formes pleines et réduites d'un même ST sont susceptibles de se côtoyer, la forme réduite précédant à l'occasion la forme pleine.

13. Pour qu'un exemple soit retenu, le lien réductionnel doit avoir été établi dans le corps d'un même texte, la réduction étant un mécanisme discursif interphrastique et, à l'occasion, intraphrastique.

14. Cette remarque vaut pour toute unité terminologique complexe de type syntagmatique, qu'elle soit synaptique (ex. satellite de télécommunications $\Longleftrightarrow$ satellite), épithétique (ex. détecteur solaire $\Longleftrightarrow$ détecteur), ou asyndétique (ex. axe lacet $\Leftrightarrow$ axe).

15. Nous jugeons qu'un rapport dialectique s'installe, dès l'actualisation du terme, entre le texte et le terme, et cela surtout sur le plan dénominationnel.

16. Par notion, nous entendons grosso modo le signifié du terme, et par dénomination son signifiant.

17. Tout comme Lotte, nous jugeons qu'il n'y a pas de changement de la signification. La préservation de la signification, cependant, nous pousse à croire que même dans le cas de la reprise anaphorique il y a changement de forme, notamment réduction de la forme pleine du ST à son noyau ou déterminé dont la forme coïncide le plus souvent avec celle du terme générique ou de l'hyperonyme.

18. Par hiérarchie onomasiologique, nous entendons les rapports d'hyperonymie, d'hyponymie et de co-hyponymie qui se tissent entre les termes d'un domaine.

19. Le syntagme plein appartenant à la terminologie d'un domaine de spécialité particulier, ses variantes réduites n'ont le statut de RL ou de RA qu'à l'intérieur de ce domaine précis.

20. C'est le dé - ou le dé du dé syntagmatique - qui détermine la catégorie grammaticale du ST.

21. Au moment d'écrire cet article, nous ne disposons pas d'exemples d'effacements d'éléments de servitude syntaxique à l'intérieur du dé.

22. L'effacement complet d'un dé complexe semble théoriquement possible, mais nous ne disposons cependant pas cncorc d'exemples. 


\section{RÉFÉRENCES}

ALBER-DEWOLF, Rosa (1984): Étude sur la création néonymique. Analyse comparêe des procédés morphologiques et morphosyntaxiques de formation des termes du domaine de la spectroscopie en anglais, en allemand, en français et en russe, Québec (Université Laval), GIRSTERM.

CNES/CNET (1982): Télécommunications spatiales. I. Bases théoriques, Paris, Masson.

CNES / CNET (1983) : Télécommunications spatiales. III. Secteur terrien. Systèmes de télécommunications par satellite, Paris, Masson.

DUQUET-PICARD, Diane (1986): La synonymie en langues de spécialité : étude du problème en terminologie, Québec (Université Laval), GIRSTERM.

FEYRY-MIANNAY, Monique (1984) : «Les groupes lexicaux complexes dans le vocabulaire de l'électronique et de l'électrotechnique», La banque des mots, $n^{\circ} 27$, pp. 31-66.

GUILBERT, Louis (1965) : La formation du vocabulaire de l' aviation, Paris, Librairie Larousse.

GUILBERT, Louis (1967) : Le vocabulaire de l'astronautique. Enquête linguistique à travers la presse d' information à l'occasion de cinq exploits de cosmonautes, Paris, Librairie Larousse.

GUILBERT, Louis (1970): «La dérivation syntagmatique dans les vocabulaires scientifiques et techniques», Les langues de spécialité. Actes du stage de Saint-Cloud, AIDELA, pp. 116-125.

GUILBERT, Louis (1975) : La créativité lexicale, Paris, Librairie Larousse.

KOCOUREK, Rostislav (1991): La langue française de la technique et de la science. Vers une linguistique de la langue savante, Wiesbaden, Oscar Brandstetter.

LOTTE, D. S. (1981): «Principes d'établissement d'une terminologie scientifique et technique», Textes choisis de terminologie. I. Fondements théoriques de la terminologie (sous la direction de V. I. Siforov), Québec (Université Laval), GIRSTERM, pp. 3-53.

MARAL, Gérard et al. (1982): Les systèmes de télécommunications par satellites, Paris, Masson.

PORTELANCE, Christine (1989): Les formations syntagmatiques en langues de spécialité, thèse de doctorat, Université de Montréal. 